\title{
Association of Duration of Sleep and Cardiovascular and Metabolic Comorbidities in Sleep Apnea Syndrome
}

\author{
Zeynep Zeren Ucar, ${ }^{1}$ Ali Kadri Cirak, ${ }^{2}$ Serhan Olcay, ${ }^{2}$ Hatice Uysal, ${ }^{2}$ \\ Ahmet Ugur Demir, ${ }^{3}$ and Rıfat Özacar ${ }^{2}$ \\ ${ }^{1}$ The Department of Sleep Disorders, Dr Suat Seren Chest Diseases and Surgery Training and Research Hospital, Yenisehir, \\ 35110 Izmir, Turkey \\ ${ }^{2}$ The Department of Pulmonary Diseases, Dr Suat Seren Chest Diseases and Surgery Training and Research Hospital, \\ 35110 Izmir, Turkey \\ ${ }^{3}$ The Department of Pulmonary Diseases, Faculty of Medicine, Hacettepe University, 06100 Ankara, Turkey
}

Correspondence should be addressed to Zeynep Zeren Ucar, zeynepzucar@yahoo.com

Received 2 October 2011; Revised 21 November 2011; Accepted 23 November 2011

Academic Editor: Sophia Schiza

Copyright ( $) 2012$ Zeynep Zeren Ucar et al. This is an open access article distributed under the Creative Commons Attribution License, which permits unrestricted use, distribution, and reproduction in any medium, provided the original work is properly cited.

\begin{abstract}
Background/Aim. Previous population-based studies found association between duration of sleep and cardiovascular and metabolic comorbidities. Our aim was to investigate the association between the duration of sleep and cardiovascular and metabolic comorbidities in OSAS. Patients and Methods. The study enrolled 312 patients, who had polysomnography (PSG) during 2006-2007 and responded to a telephone-administered questionnaire providing information on characteristics of sleep on average 12 months after PSG. Results. Of the patients, 90 were female (28.8\%), 173 (58.5) received the diagnosis of OSAS, 150 (45\%) had no comorbidities, 122 had hypertension (HT), 44 had diabetes mellitus (DM), and 38 had coronary heart disease (CHD). Mean \pm SD of age in years was 47.2 $\pm 10.6,56.5 \pm 9.3,53.2 \pm 8.9$, and $59.9 \pm 9.0$ for the no comorbidity, HT, DM, and CHD groups, respectively. Reported duration of sleep was not associated with any of the comorbidities in the overall group. In the analysis restricted to OSAS patients, sleep duration $\leq 6$ hours was significantly associated with CHD after the adjustment for age, gender, and other associated factors (OR: 5.8, 95\% CI: 1.0-32.6). Conclusions. Confirmation of the association between shorter duration of sleep and CHD will provide prognostic information and help for the management of OSAS.
\end{abstract}

\section{Introduction}

Sleep loss is a common condition in modern society. Although the health effects of sleep deprivation have been obscure, recent epidemiological studies have revealed relationships between sleep deprivation and hypertension (HT), coronary heart disease (CHD), and diabetes mellitus (DM) [1]. Because sleep deprivation increases sympathetic nervous system activity, this increased activity serves as a common pathophysiology for HT, DM, and CHD. Previous studies showed that sleep duration less than 6 hours or more than 8 hours is associated with increased morbidity and mortality due to cardiovascular diseases in the general population $[2,3]$.

Obstructive sleep apnea syndrome (OSAS) is a common medical disorder that is growing in prevalence worldwide. It is characterized by recurrent cycles of intermittent hypoxia and there is increasing evidence that intermittent hypoxia plays a role in the development of cardiovascular risk in OSAS patients through the activation of inflammatory pathways. Some excellent review articles have already summarized the effects of OSAS on HT, CHD, and DM [4-6]. The pathogenesis of cardiovascular disease in OSAS is not completely understood but is likely to be multifactorial, involving a diverse range of mechanisms including sympathetic nervous system overactivity, endothelial dysfunction, and selective activation of inflammatory molecular pathways [79]. Expanding our understanding of these pathways, which include chronic intermittent hypoxia and provocation of inflammation by sleep deprivation, will yield novel therapeutic targets with the scope of reducing the cardiovascular risk in OSAS. 


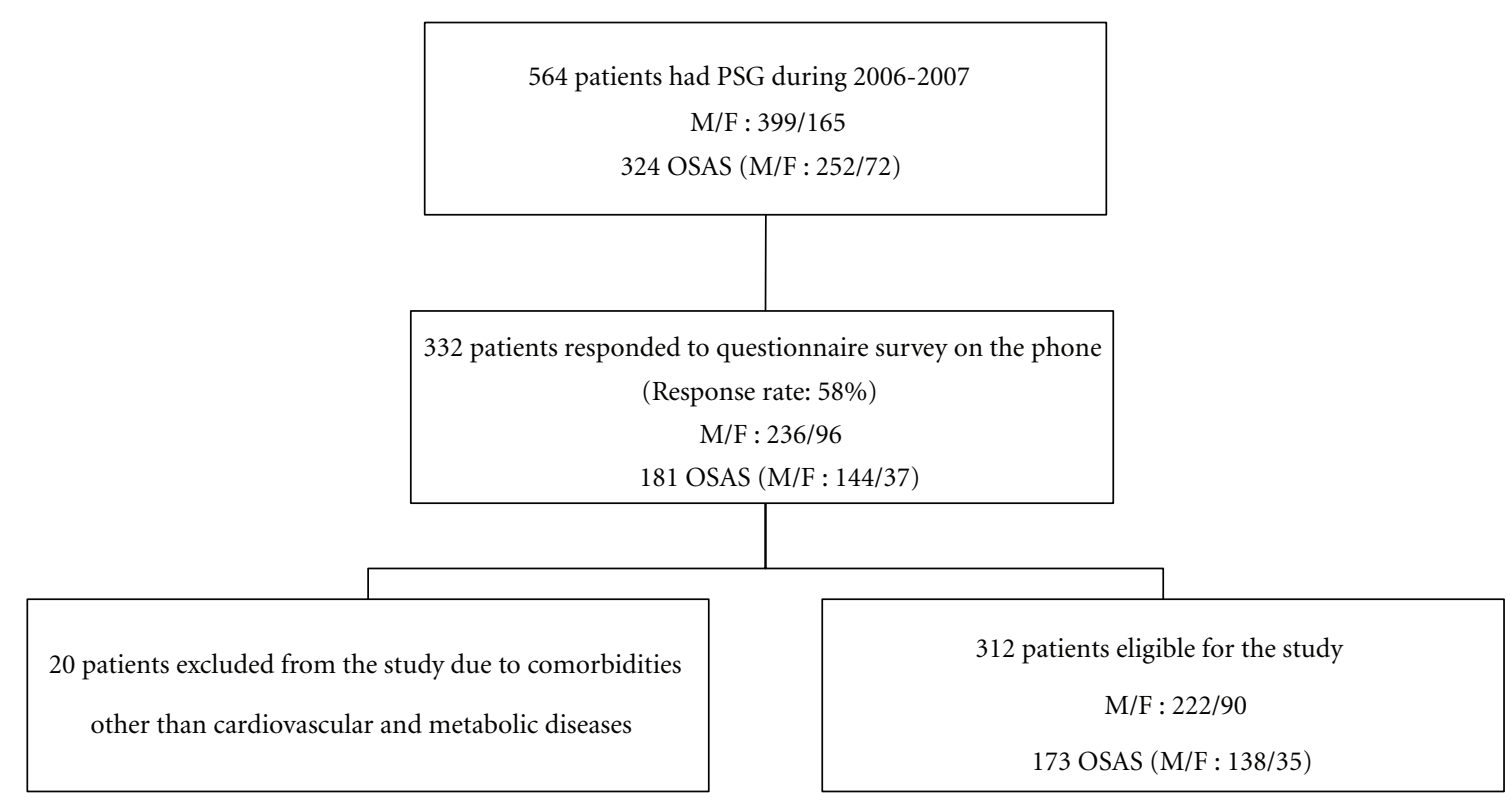

FIGURE 1: Flowchart of the study, describing the study population: PSG: polysomnography, M: male, F: female, OSAS: obstructive sleep apnea syndrome defined as excessive daytime sleepiness (Epworth Sleepiness Scale score $>10$ ) and AHI (apnea-hypopnea index) $\geq 5$ or AHI $\geq 15$, regardless of excessive daytime sleepiness.

Hence, both sleep deprivation and OSAS are related to the occurrence and consequences of cardiovascular and metabolic diseases. To our knowledge, there have been no reports on the relationship between sleep duration and risk of comorbidities in OSAS patients. Our aim was to investigate the association between duration of sleep and cardiovascular and metabolic comorbidities in patients admitted to sleep lab with sleep apnea symptoms and in patients who received the diagnosis of OSAS.

\section{Material and Methods}

2.1. Patients and Study Protocol. Of the 564 patients, who had polysomnography during 2006-2007 with a preliminary diagnosis of sleep apnea in the sleep center, 332 (response rate: 58\%) gave consent and responded to a telephone administered survey on average 12 months after polysomnography (PSG), which included information on characteristics of sleep and physician diagnosis of systemic diseases. The study protocol was approved by the Institutional Review Board of the research hospital. Cardiovascular and metabolic diseases (comorbidities) included hypertension, diabetes mellitus, and coronary heart disease. Patients were grouped in hypertension, diabetes mellitus, and coronary heart disease, and no comorbidity groups according to their responses. In the condition of reporting more than one of these diseases, the patient was grouped in all these disease groups; that is, the groups were not exclusive. Twenty patients, who reported none of these three diseases (DM, HT, and $\mathrm{CHD}$ ), but a systemic disease including neuromuscular diseases, heart failure, and chronic obstructive lung diseases were omitted from the analysis. Thus, the study population comprised of 312 subjects (55\% of the original sample).
Figure 1 describes flowchart of the study population. There was no significant difference between the age, gender, and OSAS percentage of the patients in the study population and the patients who did not respond to the telephone administered survey.

Duration of sleep was assessed by the question: "On average how many hours do you sleep at night?" In the analysis, duration of sleep was categorized as 6 hours or less, 7-8 hours, and more than 8 hours, based on the previous studies, which suggested 7 hours of sleep as normal.

2.2. Polysomnography. PSG was performed in the Sleep Laboratory with Grass Technologies Comet Series EEG/PSG with AS40 Amplifier System running Grass Technologies Twin software version 4 (Grass Technologies, Astro-Med Inc. Product Group, USA) and included four electroencephalography (EEG) channels ( $\mathrm{C} 3$ to $\mathrm{A} 1, \mathrm{C} 4$ to $\mathrm{A} 2, \mathrm{O} 1$ to $\mathrm{A} 2$, and $\mathrm{O} 2$ to A1), right and left electrooculography (EOG) channels, one chin electromyography (EMG) channel and four tibialis anterior EMG channels, finger pulse oximeter, strain gauges for thoracoabdominal movements, one electrocardiography (ECG) lead, a nasal airflow (pressure cannula), a nasal thermistor and a digital microphone for snoring detection. PSG recordings were scored in 30-second epochs for sleep, breathing and oxygenation according to the standard criteria of the American Academy of Sleep Medicine (AASM) [10]. Obstructive apnea was defined as a $90 \%$ cessation of oronasal airflow for at least 10 seconds in the presence of chest-wall motion. Hypopnea was defined as a reduction in the airflow of $50 \%$ or more associated with $3 \%$ or more arterial oxygen desaturation and/or arousal, or a reduction in respiratory airflow of $30 \%$ or more associated with $4 \%$ or more arterial oxygen desaturation and/or arousal for at least 
TABLE 1: Demographic features of the patients.

\begin{tabular}{|c|c|c|c|c|c|}
\hline & $N$ & No comorbidity & HT & $\mathrm{DM}$ & $\mathrm{CHD}$ \\
\hline$N$ & 312 & 150 & 122 & 44 & 38 \\
\hline Male/Female, (\%) & $222 / 90$ & $116 / 34(77.3) /(22.7)$ & $76 / 46^{* *}(62.3) /(37.7)$ & $29 / 15(63.4) /(36.6)$ & $28 / 10(74.3) /(25.7)$ \\
\hline Age, (year) & & $47.2 \pm 10.6$ & $56.5 \pm 9.3^{* * *}$ & $53.2 \pm 8.9^{* *}$ & $59.9 \pm 9.0^{* * *}$ \\
\hline BMI, $\left(\mathrm{kg} / \mathrm{m}^{2}\right)$ & & $30.0 \pm 4.8$ & $32.5 \pm 6.0^{* * *}$ & $32.3 \pm 5.9^{*}$ & $30.6 \pm 4.8$ \\
\hline \multicolumn{6}{|l|}{ Educational status } \\
\hline Primary or less, $\%$ & 149 & 43.9 & 51.3 & 47.5 & $82.4^{* * *}$ \\
\hline Secondary, \% & 98 & 36.6 & 31.9 & 27.5 & 11.8 \\
\hline University, \% & 59 & 19.6 & 16.8 & 25.0 & 5.9 \\
\hline \multicolumn{6}{|l|}{ Smoking status } \\
\hline Nonsmoker, \% & 120 & 36.7 & 46.3 & 36.6 & 31.4 \\
\hline Ex-smoker, \% & 94 & 34.7 & 24.0 & 36.6 & 20.0 \\
\hline Current smoker, \% & 97 & 28.7 & 29.8 & 26.8 & 48.6 \\
\hline Shift work, \% & 73 & 23.3 & 21.7 & 31.7 & 25.7 \\
\hline
\end{tabular}

Means and \pm SDs are given in the table, unless otherwise specified.

$* P<0.05$;

$* * P<0.01$

$* * * P<0.001$.

Significant findings are marked in bold type.

HT: hypertension, DM: diabetes Mellitus, CHD: coronary heart disease, and OSAS: obstructive sleep apnea syndrome.

10 seconds. Apnea hypopnea index (AHI) was calculated as the total number of apneas and hypopneas per hour of sleep. Diagnosis of OSAS was based on PSG findings, according to the International Classification of Sleep Disorders 2 (ICSD2) [11], as in the following:

(i) Excessive daytime sleepiness and $\mathrm{AHI} \geq 5$;

(ii) $\mathrm{AHI} \geq 15$, regardless of excessive daytime sleepiness.

Excessive daytime sleepiness was defined as the score of Epworth sleepiness scale $>10$ [12]. Subjects were classified in the control group if they had AHI $<5$ regardless of excessive daytime sleepiness.

2.3. Statistical Analysis. Descriptive statistics and comparison of the comorbidity groups with the no comorbidity group are shown in the tables. Frequency (percentage) and mean \pm standard deviation were used for the descriptive statistics of the categorical and continuous variables, respectively. Chi-square testing was used for the univariate analysis of categorical variables. Fisher's exact test was used in the case of an expected frequency of less than five in $25 \%$ of the cells. Student's $t$-testing and Mann-Whitney $U$ test were used for the univariate analysis of continuous variables, where appropriate (normal and not-normal distribution, resp.). Logistic regression analysis models were constructed to adjust the association between duration of sleep and comorbidities for age, gender, BMI, other potential risk factors, and factors which were found significant in the univariate analysis. Models were constructed both in the overall group and in the OSAS group, to investigate the association between duration of sleep and comorbidities both in the overall study population and in OSAS patients. $P$ value less than 0.05 was used to define statistical significance in the two-sided tests.

\section{Results}

The study enrolled 312 patients, who had PSG with a preliminary diagnosis of sleep apnea between 2006 and 2007 and answered a telephone administered questionnaire providing information on characteristics of sleep. Table 1 shows the personal characteristics of the subjects in the cardiovascular and metabolic comorbidity groups. Of the subjects 90 were female $(28.8 \%), 150(45 \%)$ had no comorbidity, 122 had HT (39.1\%), 44 had DM (14.1\%), and 38 had CHD (12.1\%). Diagnosis of OSAS was made in $173(58.5 \%)$ of the patients, without a significant difference between the groups of HT (60.2\%), DM (48.7\%), and CHD (48.4\%) and no comorbidity group (58.5\%). Of the OSAS patients 138 were men and 35 were women. Coronary heart disease group compared to no comorbidity group had a higher percentage of less educated patients. In the PSG record, time percentage spent $<90 \%$ of oxygen (T90\%) was significantly higher in the HT group than no comorbidity group. Table 2 shows the association between PSG findings and cardiovascular and metabolic comorbidity groups.

Of the patients 58 reported onset of sleep delayed (delayed sleep onset) more than 30 minutes (18.5\%), 211 had fragmented sleep (67.6\%), 174 had unrefreshening sleep (55.7\%), and 78 had excessive daytime sleepiness defined as ESS score above $10(25 \%)$. There was no significant difference in the prevalence of sleep symptoms between comorbidity groups and no comorbidity group, except for the significantly higher percentage of delayed sleep onset in the HT group than the no comorbidity group $(25.4 \%$ versus 13.3).

Duration of sleep was reported as 6 hours or less in 15\%, $>6$ hours to 7 hours in $25.2 \%,>7$ hours to 8 hours in $47.9 \%$, and $>8$ hours in $11.8 \%$ of the subjects. There was no significant difference between the sleep duration of the 
TABLE 2: Polysomnography findings and features of OSAS of the patients in different cardiovascular and metabolic comorbidity groups.

\begin{tabular}{|c|c|c|c|c|c|}
\hline & $N$ & No comorbidity & HT & $\mathrm{DM}$ & $\mathrm{CHD}$ \\
\hline$N$ & 312 & 150 & 122 & 44 & 38 \\
\hline Total sleep time, min & & $385.4 \pm 82.1$ & $346.5 \pm 88.6^{* *}$ & $335.8 \pm 87.0^{* *}$ & $348.5 \pm 98.9$ \\
\hline T90\%, (\%) & & $13.4 \pm 21.8$ & $22.8 \pm 30.8^{*}$ & $23.6 \pm 5.0$ & $16.2 \pm 26.8$ \\
\hline Sleep efficiency, (\%) & & $87.5 \pm 10.5$ & $84.5 \pm 10.5^{*}$ & $84.0 \pm 13.3$ & $84.8 \pm 11.4$ \\
\hline WASO, (\%) & & $2.2 \pm 6.3$ & $4.3 \pm 8.4^{*}$ & $4.0 \pm 9.1$ & $3.5 \pm 7.6$ \\
\hline REM, (\%) & & $11.3 \pm 6.4$ & $9.5 \pm 7.1^{*}$ & $10.4 \pm 5.8$ & $11.3 \pm 7.4$ \\
\hline N3, (\%) & & $21.8 \pm 12.4$ & $21.0 \pm 14.6$ & $16.4 \pm 5.8^{*}$ & $20.0 \pm 15.0$ \\
\hline AHI, (/hour) & & $30.3 \pm 27.9$ & $33.1 \pm 30.7$ & $31.3 \pm 31.5$ & $22.4 \pm 22.2$ \\
\hline Obstructive AHI & & $27.6 \pm 26.4$ & $31.9 \pm 30.1$ & $27.1 \pm 27.9$ & $19.9 \pm 19.0$ \\
\hline OSAS, $\%$ & 173 & 58.5 & 60.2 & 48.7 & 48.4 \\
\hline Severe OSAS, \% & 120 & 38.5 & 38.0 & 34.1 & 27.8 \\
\hline CPAP prescribed, $\%$ & 116 & 33.3 & 37.7 & 48.8 & 38.9 \\
\hline CPAP compliance, $\%$ & 61 & 52.0 & 55.6 & 60.0 & 42.9 \\
\hline
\end{tabular}

Means and \pm SDs are given in the table, unless otherwise specified.

${ }^{*} P<0.05$;

$* * P<0.01$

Significant findings are marked in bold type.

HT: hypertension, DM: diabetes mellitus, CHD: coronary heart disease, ESS: Epworth Sleepiness Scale, OSAS: obstructive sleep apnea syndrome, and WASO: wake after sleep onset.

OSAS: excessive daytime sleepiness and $\mathrm{AHI} \geq 5$ or $\mathrm{AHI} \geq 15$, regardless of excessive daytime sleepiness.

Severe OSAS was defined as AHI above $30 / \mathrm{h}$.

CPAP compliance: reported usage of CPAP every night for more than 4 hours a night. CPAP compliance percentage was calculated in the patients who were prescribed CPAP therapy.

TABLE 3: Association between reported sleep duration and cardiovascular and metabolic comorbidities in the models adjusting for other risk factors.

Whole group

OSAS patients

\begin{tabular}{|c|c|c|c|c|}
\hline $\begin{array}{l}\text { Comorbidities and duration } \\
\text { of sleep }\end{array}$ & Unadjusted OR (95\% CI) & Adjusted OR (95\% CI) & Unadjusted OR (95\% CI) & Adjusted OR (95\% CI) \\
\hline \multicolumn{5}{|l|}{ HT } \\
\hline 6 hours or less & $1.0(0.6-1.8)$ & $0.6(0.3-1.3)$ & $1.3(0.6-2.7)$ & $0.7(0.2-1.7)$ \\
\hline 8.5-10 hours & $1.0(0.4-2.2)$ & $0.7(0.3-1.8)$ & $1.4(0.5-4.4)$ & $0.8(0.2-2.7)$ \\
\hline \multicolumn{5}{|l|}{$\mathrm{DM}$} \\
\hline 6 hours or less & $1.6(0.7-3.5)$ & $1.2(0.5-3.0)$ & $2.7(0.8-8.5)$ & $2.3(0.7-7.8)$ \\
\hline 8.5-10 hours & $1.7(0.6-4.8)$ & $1.2(0.4-3.7)$ & $3.7(0.9-15.9)$ & $2.3(0.4-11.4)$ \\
\hline \multicolumn{5}{|l|}{$\mathrm{CHD}$} \\
\hline 6 hours or less & $1.1(0.5-2.9)$ & $1.2(0.4-3.8)$ & $3.6(1.1-11.3)$ & $5.8(1.0-32.6)$ \\
\hline 8.5-10 hours & $0.4(0.1-2.1)$ & $0.3(0.06-2.3)$ & NA & NA \\
\hline
\end{tabular}

Adjustments were made for OSAS, age, gender, and BMI in all models, and additionally for smoking status in HT and for smoking status and educational status in CHD.

For duration of sleep, 7-8 hours was considered as the reference category.

Significant findings are marked in bold type.

HT: hypertension, DM: diabetes mellitus, CHD: coronary heart disease, and OSAS: obstructive sleep apnea syndrome.

comorbidity groups and the no comorbidity group. Figure 2 shows the boxplot graph of the distribution of duration of sleep in HT, DM, and CHD groups compared to no comorbidity group in patients with and without OSAS. Among the OSAS patients, duration of sleep was significantly shorter in the CHD group than in the no comorbidity group.

Table 3 shows the association between reported sleep duration and cardiovascular and metabolic comorbidity groups in the models adjusting for other risk factors. In the whole group duration of sleep was not associated with any of the comorbidities. In the analysis restricted to OSAS patients, sleep duration of $\leq 6$ hours was significantly associated with coronary heart disease as unadjusted and after the adjustment for age, gender, smoking status, and educational status.

\section{Discussion}

In this study, we have demonstrated that shorter duration of sleep (6 hours or less) was associated with CHD in 


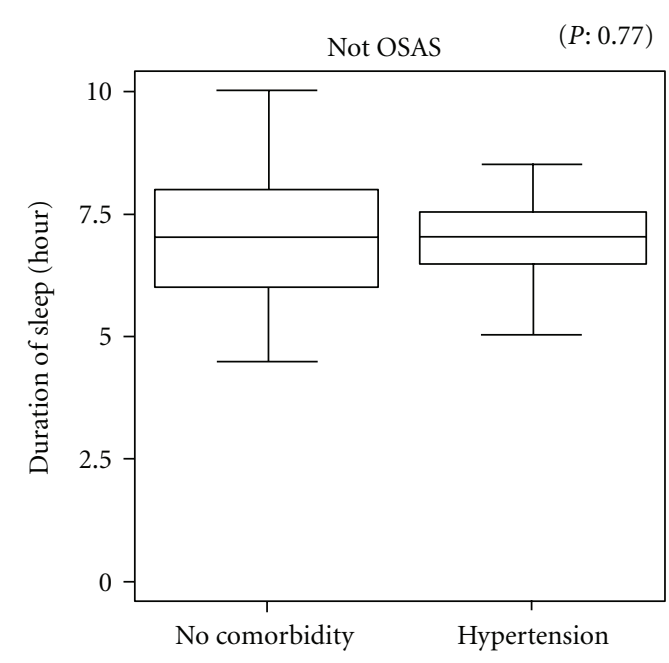

(a)

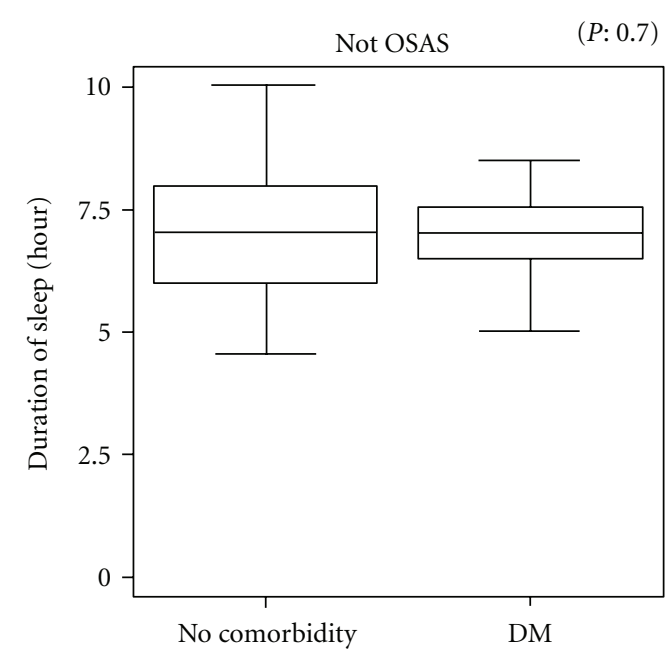

(c)

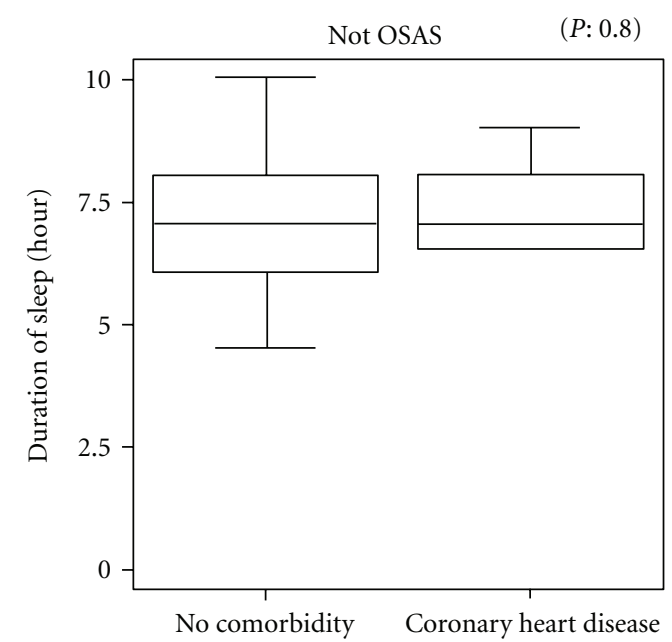

(e)

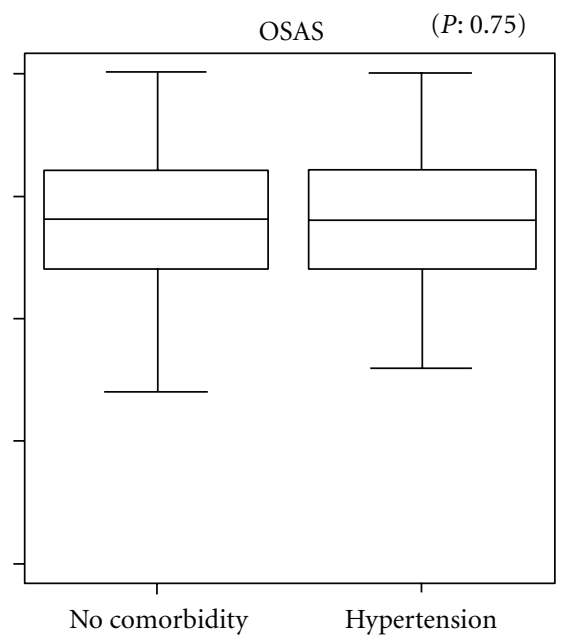

(b)

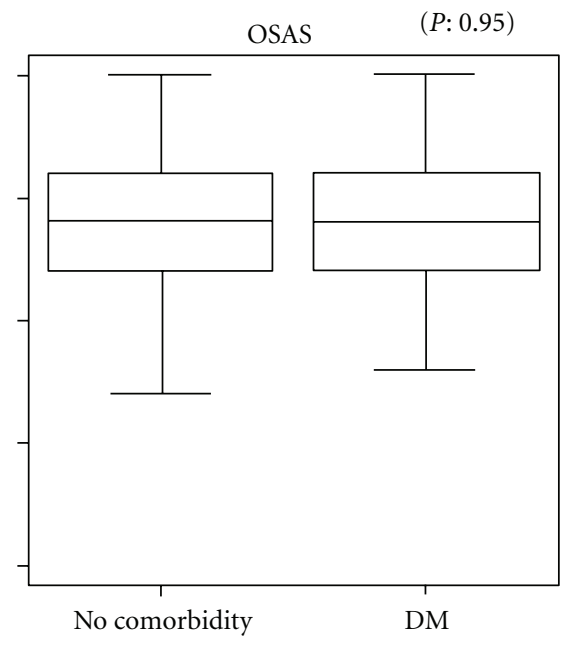

(d)

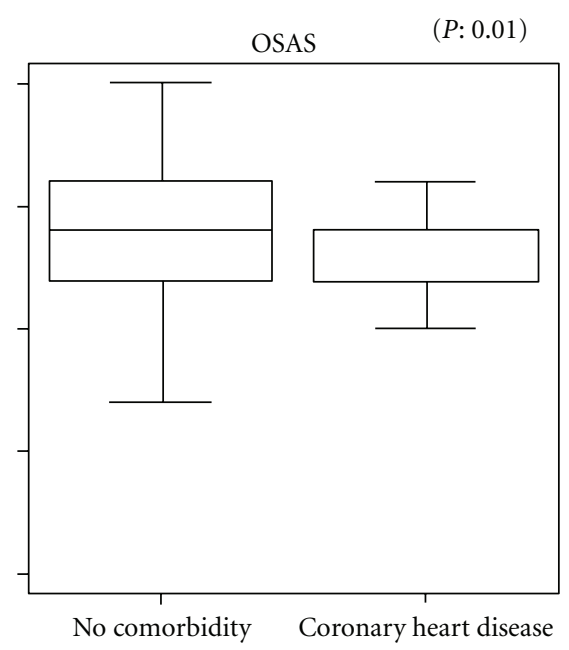

(f)

FIGURE 2: Boxplot graph of the distribution of duration of sleep in HT, DM, and CHD comorbidity groups compared to no comorbidity group in the subjects without OSAS (Not-OSAS) in graphs a, $\mathrm{c}$ and e and in the subjects with OSAS (OSAS) in graphs b, d and f: HT: hypertension, DM: diabetes mellitus, CHD: coronary heart disease, and OSAS: obstructive sleep apnea syndrome. Mann-Whitney $U$ test was used in the comparisons. 
patients with OSAS after adjustment for confounding factors in patients with OSAS.

To our knowledge, this is the first study which examined the association between sleep duration and cardiovascular and metabolic comorbidities in patients with OSAS. Previous studies found significant association between short (less than 6 hours) and long sleep duration (more than 8 hours) and CHD, DM, and HT in general population $[2,3]$.

Recent studies including follow-up data have shown that OSAS has a causal relationship with the development of cardiovascular disease independent of confounding factors such as sex, age, and obesity [13-22]. The mechanisms by which OSAS exerts its detrimental effects remain to be established, and future studies should actively pursue the identification of OSAS patients at high risk of cardiovascular diseases. Epidemiological studies have convincingly shown that type 2 diabetes is often associated with OSAS and daytime sleepiness [23-25]. For example, in a large series of OSAS patients, type 2 diabetes and impaired glucose tolerance showed a 30 and $20 \%$ prevalence, respectively [26], and studies in snorers reached similar conclusions $[27,28]$. There is strong evidence that OSAS is an independent risk factor for systemic hypertension [29, 30]. Case-control studies have confirmed the association between sleep apnea and increased blood pressure independent of confounders such as obesity [31]. Severity of OSAS (AHI, ESS, and T90\%) was not associated with DM and CHD in our study. Percentage of night time spent with oxyhemoglobin saturation below $90 \%$ was higher, and difficulty in initiating sleep was more frequent in patients with HT.

The pathogenesis of cardiovascular disease in OSAS is not completely understood but likely to be multifactorial, involving a diverse range of mechanisms including sympathetic nervous system overactivity, selective activation of inflammatory pathways, endothelial dysfunction, hypercoagulability, and metabolic dysregulation, the latter particularly involving insulin resistance and disordered lipid metabolism. Such a clinical context makes it difficult to assess the independent effects of OSAS on cardiovascular risk, and still there remains factors to be understood [32, 33].

The literature supports the view that short or long sleep duration is independently associated with an increased likelihood of coronary events [2, 34-39]. Increased unadjusted CHD risk was found in both short sleeper groups (extremely short $\leq 5 \mathrm{~h}$, and $6 \mathrm{~h}$ sleepers) and extremely long sleepers $(\geq 10 \mathrm{~h})$ in both genders when compared with midrange (7$8 \mathrm{~h}$ ) sleepers. It was also reported as an important finding that 9-hour sleepers did not differ in their CHD risk when compared with midrange sleepers in the same study [40]. Short sleep duration $(<6 \mathrm{~h})$ has been shown as a significant risk factor for coronary events in a Japanese male working population [41]. The risk of CHD events was independent of prominent cardiovascular risk factors and occupational factors [41]. Short sleep duration imposed on a group of healthy subjects increased sympathetic nervous system activity and blood pressure elevation. Therefore, sustained short sleep duration could lead to adverse cardiovascular consequence. We investigated the association between reported sleep duration and cardiovascular and metabolic comorbidities in the models adjusting for the other risk factors including obesity and smoking [42-44]. In these models, only CHD in OSAS patients was associated with shorter sleep duration. Since a few subjects reported sleep duration of 10 hours or more, we could not investigate the association between longer sleep time and CHD.

The strengths of the study were examination of both PSG data and self-reported sleep duration and their association with comorbidities. We defined no comorbidity group as patients who did not report the investigated diseases and other systemic diseases. However, these patients might have comorbidities (which were not diagnosed yet), and this misclassification, if nondifferential, could be underestimating the association between sleep duration and comorbidities.

The present study had some limitations. The major limitations of the study were small sample size, which was a constraint for the adjustment of the potential confounders in the analysis. Since this is a cross-sectional study it is difficult to interpret the causality of our findings. However, there is not enough reason to believe that comorbidities strongly affected the duration of sleep. Sleep duration was only associated with CHD in the OSAS group. If comorbidities had strongly changed the sleep duration, this association must have also been observed in DM and HT. We did not have data about the other confounders like hyperlipidemia, alcohol intake, and exercise frequency and therefore could not control these factors in the analysis confounders. Objective measurements of sleep duration, blood pressure, and glucose were lacking, which is another limitation of our study. The prevalence of comorbidities in the control group was not significantly different from the patients with OSAS, which suggests that selection bias (i.e., higher degree of suspicion and screening for systemic diseases and thus finding higher prevalence of systemic diseases in OSAS patients) is not so likely. In this study, reported sleep duration and sleep symptoms were used as an estimate of the general status of sleep habits and sleep symptoms. Patients who were using CPAP therapy were asked to report the situation (duration of sleep and sleep symptoms) before treatment. We did not find a beneficial effect of CPAP compliance on the sleep-related symptoms, which could be due to questions asking the subjects about their symptoms before treatment. Duration of sleep and quality of sleep might have changed due to comorbidities, and this could influence the interpretation of the associations found in this study. Lack of association between sleep duration and other comorbidities (HT and DM) could be regarded as evidence against this possibility. Importantly, the control group consisted of patients who were admitted to the sleep center with sleep-related complaints, including snoring and poor quality of sleep. Previous studies which used snoring as a proxy for sleep-related breathing disorders have found significant association between snoring and cardiovascular diseases $[45,46]$. Thus, the control group in this study was not representing the healthy individuals in the general population. This could, at least partially explain the lack of association between comorbidities and duration of sleep and OSAS, in the study population. Presence of sleep symptoms (excessive daytime sleepiness, unrefreshening sleep, or difficulty in initiating sleep) was not 
associated with the comorbidities in the regression analysis. This could be due to the high prevalence of these symptoms in the no comorbidity group (which was almost similar to that of comorbidity groups). The only significant association was found between CHD and duration of sleep in the analysis restricted to OSAS patients. This could suggest the interaction between OSAS and short duration of sleep for the development of CHD. Confirmation of this finding requires follow-up data.

In conclusion, CHD was associated with shorter sleep duration in patients with OSAS in the present study. Our results, if confirmed, suggest that ensuring appropriate sleep hours through appropriate OSAS treatment and modifying lifestyle factors and sleep hygiene could be beneficial in the prevention of cardiovascular and coronary events in patients with OSAS.

\section{References}

[1] T. G. Pickering, "Could hypertension be a consequence of the 24/7 society? The effects of sleep deprivation and shift work," Journal of Clinical Hypertension, vol. 8, no. 11, pp. 819-822, 2006.

[2] D. L. Wingard and L. F. Berkman, "Mortality risk associated with sleeping patterns among adults," Sleep, vol. 6, no. 2, pp. 102-107, 1983.

[3] F. P. Cappuccio, D. Cooper, L. Delia, P. Strazzullo, and M. A. Miller, "Sleep duration predicts cardiovascular outcomes: a systematic review and meta-analysis of prospective studies," European Heart Journal, vol. 32, no. 12, pp. 1484-1492, 2011.

[4] R. Wolk, A. S. M. Shamsuzzaman, and V. K. Somers, "Obesity, sleep apnea, and hypertension," Hypertension, vol. 42, no. 6, pp. 1067-1074, 2003.

[5] R. S. T. Leung and T. D. Bradley, "Sleep apnea and cardiovascular disease," American Journal of Respiratory and Critical Care Medicine, vol. 164, no. 12, pp. 2147-2165, 2002.

[6] N. M. Punjabi and V. Y. Polotsky, "Disorders of glucose metabolism in sleep apnea," Journal of Applied Physiology, vol. 99, no. 5, pp. 1998-2007, 2005.

[7] W. T. McNicholas and M. R. Bonsignore, "Sleep apnoea as an independent risk for cardiovascular disease: current evidence, basic mechanisms and research priorities," European Respiratory Journal, vol. 29, no. 1, pp. 156-178, 2007.

[8] S. M. Caples, A. S. Gami, and V. K. Somers, "Obstructive sleep apnea," Annals of Internal Medicine, vol. 142, no. 3, pp. 187197, 2005.

[9] A. Williams and S. M. Scharf, "Obstructive sleep apnea, cardiovascular disease, and inflammation-is NF- $\kappa$ B the key?" Sleep and Breathing, vol. 11, no. 2, pp. 69-76, 2007.

[10] C. Iber, S. Ancoli-Israel, A. L. Chesson, and S. F. Quan, The AASM Manual for the Scoring of Sleep and Associated Events: Rules, Terminology, and Technical Specifications, Westchester, Ill, USA, 2007.

[11] The International Classification of Sleep Disorders: Diagnostic and Coding Manual, Westchester, Ill, USA, 2nd edition, 2005.

[12] M. W. Johns, "Sleepiness in different situations measured by the Epworth Sleepiness Scale," Sleep, vol. 17, no. 8, pp. 703710, 1994.

[13] N. S. Marshall, K. K. H. Wong, P. Y. Liu, S. R. J. Cullen, M. W. Knuiman, and R. R. Grunstein, "Sleep apnea as an independent risk factor for all-cause mortality: the Busselton Health Study," Sleep, vol. 31, no. 8, pp. 1079-1085, 2008.
[14] T. Young, L. Finn, P. E. Peppard et al., "Sleep disordered breathing and mortality: eighteen-year follow-up of the Wisconsin sleep cohort," Sleep, vol. 31, no. 8, pp. 1071-1078, 2008.

[15] F. J. Nieto, T. B. Young, B. K. Lind et al., "Association of sleepdisordered breathing sleep apnea, and hypertension in a large community-based study. Sleep Heart Health Study," Journal of the American Medical Association, vol. 283, no. 14, pp. 18291836, 2000.

[16] E. O. Bixler, A. N. Vgontzas, H. M. Lin et al., "Association of hypertension and sleep-disordered breathing," Archives of Internal Medicine, vol. 160, no. 15, pp. 2289-2295, 2000.

[17] M. Partinen and C. Guilleminault, "Daytime sleepiness and vascular morbidity at seven-year follow-up in obstructive sleep apnea patients," Chest, vol. 97, no. 1, pp. 27-32, 1990.

[18] S. T. Pendlebury, J. L. Pépin, D. Veale, and P. Lévy, "Natural evolution of moderate sleep apnoea syndrome: significant progression over a mean of 17 months," Thorax, vol. 52, no. 10, pp. 872-878, 1997.

[19] A. Zaninelli, R. Fariello, E. Boni, L. Corda, C. Alicandri, and V. Grassi, "Snoring and risk of cardiovascular disease," International Journal of Cardiology, vol. 32, no. 3, pp. 347-351, 1991.

[20] Y. Peker, J. Hedner, J. Norum, H. Kraiczi, and J. Carlson, "Increased incidence of cardiovascular disease in middle-aged men with obstructive sleep apnea: a 7-year follow-up," American Journal of Respiratory and Critical Care Medicine, vol. 166, no. 2, pp. 159-165, 2002.

[21] J. M. Marin, S. J. Carrizo, E. Vicente, and A. G. N. Agusti, "Long-term cardiovascular outcomes in men with obstructive sleep apnoea-hypopnoea with or without treatment with continuous positive airway pressure: an observational study," The Lancet, vol. 365, no. 9464, pp. 1046-1053, 2005.

[22] L. S. Doherty, J. L. Kiely, V. Swan, and W. T. McNicholas, "Long-term effects of nasal continuous positive airway pressure therapy on cardiovascular outcomes in sleep apnea syndrome," Chest, vol. 127, no. 6, pp. 2076-2084, 2005.

[23] N. M. Punjabi, M. M. Ahmed, V. Y. Polotsky, B. A. Beamer, and C. P. O'Donnell, "Sleep-disordered breathing, glucose intolerance, and insulin resistance," Respiratory Physiology and Neurobiology, vol. 136, no. 2-3, pp. 167-178, 2003.

[24] M. S. M. Ip, B. Lam, M. M. T. Ng, W. K. Lam, K. W. T. Tsang, and K. S. L. Lam, "Obstructive sleep apnea is independently associated with insulin resistance," American Journal of Respiratory and Critical Care Medicine, vol. 165, no. 5, pp. 670-676, 2002.

[25] N. M. Punjabi, E. Shahar, S. Redline, D. J. Gottlieb, R. Givelber, and H. E. Resnick, "Sleep-disordered breathing, glucose intolerance, and insulin resistance: the Sleep Heart Health Study," American Journal of Epidemiology, vol. 160, no. 6, pp. 521-530, 2004.

[26] N. Meslier, F. Gagnadoux, P. Giraud et al., "Impaired glucoseinsulin metabolism in males with obstructive sleep apnoea syndrome," European Respiratory Journal, vol. 22, no. 1, pp. 156-160, 2003.

[27] P. L. Enright, A. B. Newman, P. W. Wahl, T. A. Manolio, E. F. Haponik, and P. J. R. Boyle, "Prevalence and correlates of snoring and observed apneas in 5,201 older adults," Sleep, vol. 19, no. 7, pp. 531-538, 1996.

[28] W. K. Al-Delaimy, J. E. Manson, W. C. Willett, M. J. Stampfer, and F. B. Hu, "Snoring as a risk factor for type II diabetes mellitus: a prospective study," American Journal of Epidemiology, vol. 155, no. 5, pp. 387-393, 2002.

[29] G. V. Robinson, J. R. Stradling, and R. J. O. Davies, "Sleep · 6: obstructive sleep apnoea/hypopnoea syndrome and hypertension," Thorax, vol. 59, no. 12, pp. 1089-1094, 2004. 
[30] R. A. Dart, J. R. Gregoire, D. D. Gutterman, and S. H. Woolf, "The association of hypertension and secondary cardiovascular disease with sleep-disordered breathing," Chest, vol. 123, no. 1, pp. 244-260, 2003.

[31] C. W. H. Davies, J. H. Crosby, R. L. Mullins, C. Barbour, R. J. O. Davies, and J. R. Stradling, "Case-control study of 24 hour ambulatory blood pressure in patients with obstructive sleep apnoea and normal matched control subjects," Thorax, vol. 55, no. 9, pp. 736-740, 2000.

[32] J. L. Kiely and W. T. McNicholas, "Cardiovascular risk factors in patients with obstructive sleep apnoea syndrome," European Respiratory Journal, vol. 16, no. 1, pp. 128-133, 2000.

[33] S. R. Coughlin, L. Mawdsley, J. A. Mugarza, P. M. A. Calverley, and J. P. H. Wilding, "Obstructive sleep apnoea is independently associated with an increased prevalence of metabolic syndrome," European Heart Journal, vol. 25, no. 9, pp. 735$741,2004$.

[34] D. F. Kripke, R. N. Simons, L. Garfinkel, and E. C. Hammond, "Short and long sleep and sleeping pills. Is increased mortality associated?" Archives of General Psychiatry, vol. 36, no. 1, pp. 103-116, 1979.

[35] M. Partinen, P. T. S. Putkonen, J. Kaprio, M. Koskenvuo, and I. Hilakivi, "Sleep disorders in relation to coronary heart disease," Acta Medica Scandinavica, vol. 212, supplement 660, pp. 69-83, 1982.

[36] Y. Liu, H. Tanaka, H. Kodama et al., "Overtime work, insufficient sleep, and risk of non-fatal acute myocardial infarction in Japanese men," Occupational and Environmental Medicine, vol. 59, no. 7, pp. 447-451, 2002.

[37] N. T. Ayas, D. P. White, J. E. Manson et al., "A prospective study of sleep duration and coronary heart disease in women," Archives of Internal Medicine, vol. 163, no. 2, pp. 205-209, 2003.

[38] C. Meisinger, M. Heier, H. Löwel, A. Schneider, and A. Döring, "Sleep duration and sleep complaints and risk of myocardial infarction in middle-aged men and women from the general population: the MONICA/KORA Augsburg cohort study," Sleep, vol. 30, no. 9, pp. 1121-1127, 2007.

[39] Y. Amagai, S. Ishikawa, T. Gotoh, K. Kayaba, Y. Nakamura, and E. Kajii, "Sleep duration and incidence of cardiovascular events in a Japanese population: the Jichi Medical School cohort study," Journal of Epidemiology, vol. 20, no. 2, pp. 106$110,2010$.

[40] E. Kronholm, T. Laatikainen, M. Peltonen, R. Sippola, and T. Partonen, "Self-reported sleep duration, all-cause mortality, cardiovascular mortality and morbidity in Finland," Sleep Medicine, vol. 12, no. 3, pp. 215-221, 2011.

[41] Y. Hamazaki, Y. Morikawa, K. Nakamura et al., "The effects of sleep duration on the incidence of cardiovascular events among middle-aged male workers in Japan," Scandinavian Journal of Work, Environment and Health, vol. 37, no. 5, pp. 411-417, 2011.

[42] N. M. Al Lawati, S. R. Patel, and N. T. Ayas, "Epidemiology, risk factors, and consequences of obstructive sleep apnea and short sleep duration," Progress in Cardiovascular Diseases, vol. 51, no. 4, pp. 285-293, 2009.

[43] W. B. Borden and M. H. Davidson, "Updating the assessment of cardiac risk: beyond Framingham," Reviews in Cardiovascular Medicine, vol. 10, no. 2, pp. 63-71, 2009.

[44] M. Dochi, K. Sakata, M. Oishi, K. Tanaka, E. Kobayashi, and Y. Suwazono, "Smoking as an independent risk factor for hypertension: a 14-year longitudinal study in male Japanese workers," Tohoku Journal of Experimental Medicine, vol. 217, no. 1, pp. 37-43, 2009.
[45] W. W. Schmidt-Nowara, D. B. Coultas, C. Wiggins, B. E. Skipper, and J. M. Samet, "Snoring in a Hispanic-American population: risk factors and association with hypertension and other morbidity," Archives of Internal Medicine, vol. 150, no. 3, pp. 597-601, 1990.

[46] R. D’Alessandro, C. Magelli, G. Gamberini et al., "Snoring every night as a risk factor for myocardial infarction: a casecontrol study," British Medical Journal, vol. 300, no. 6739, pp. 1557-1558, 1990. 


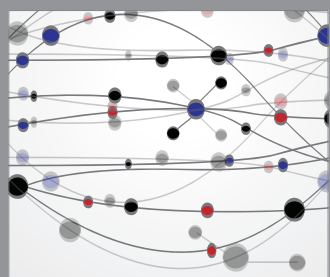

The Scientific World Journal
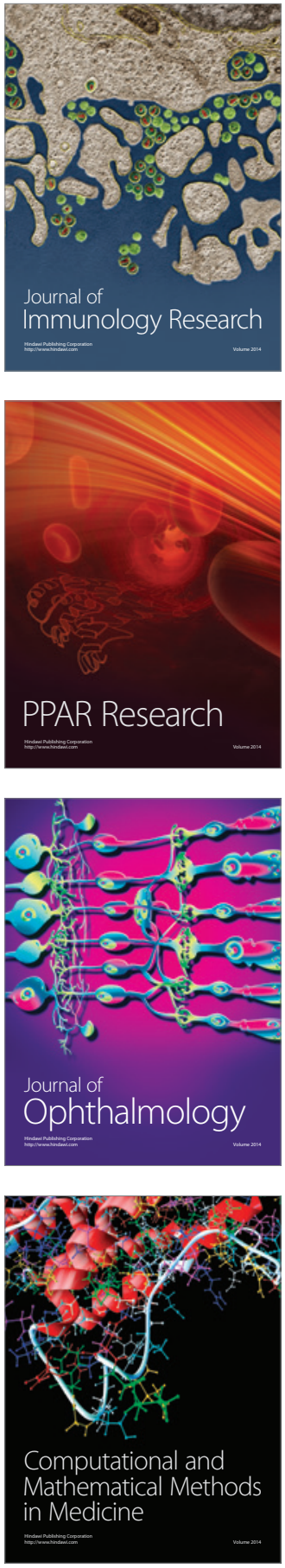

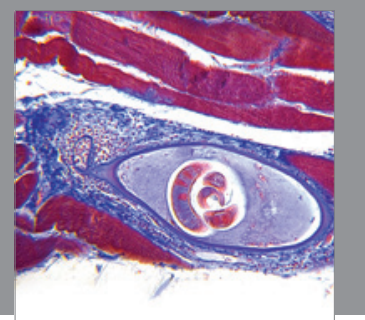

Gastroenterology

Research and Practice
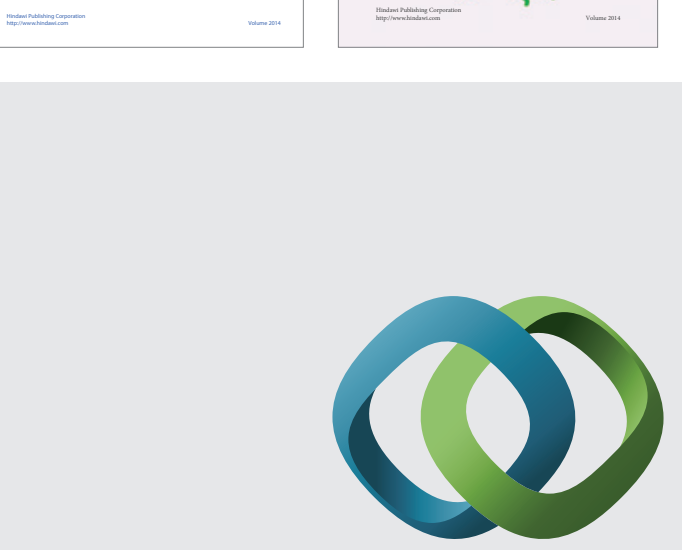

\section{Hindawi}

Submit your manuscripts at

http://www.hindawi.com
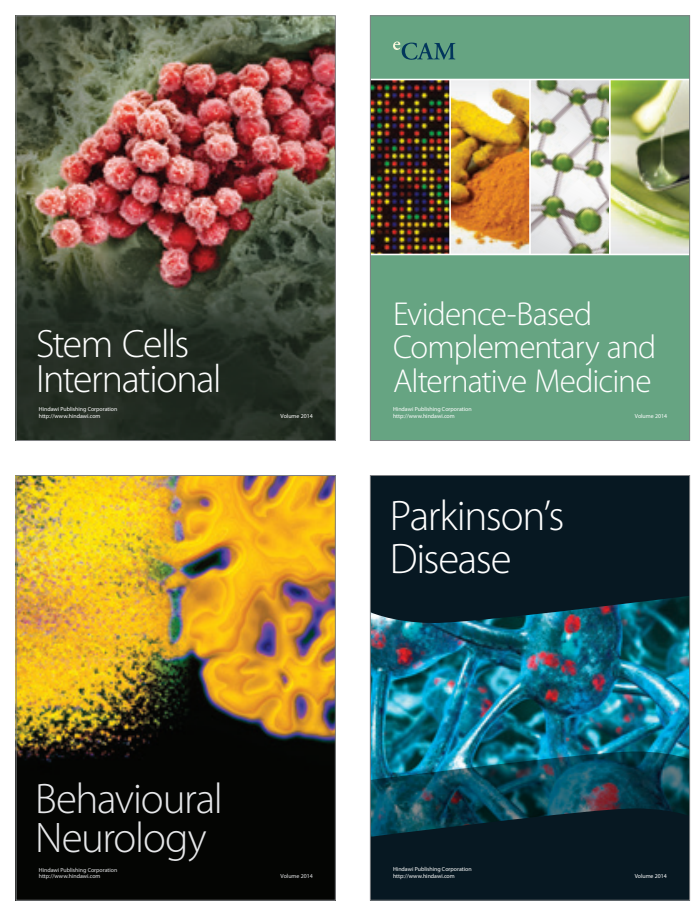

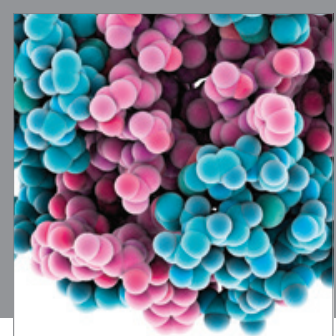

Journal of
Diabetes Research

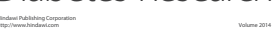

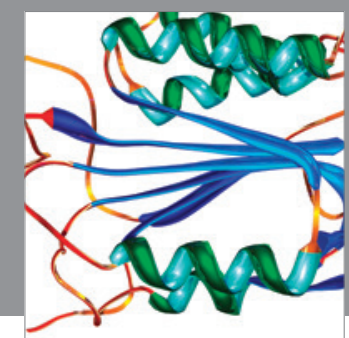

Disease Markers
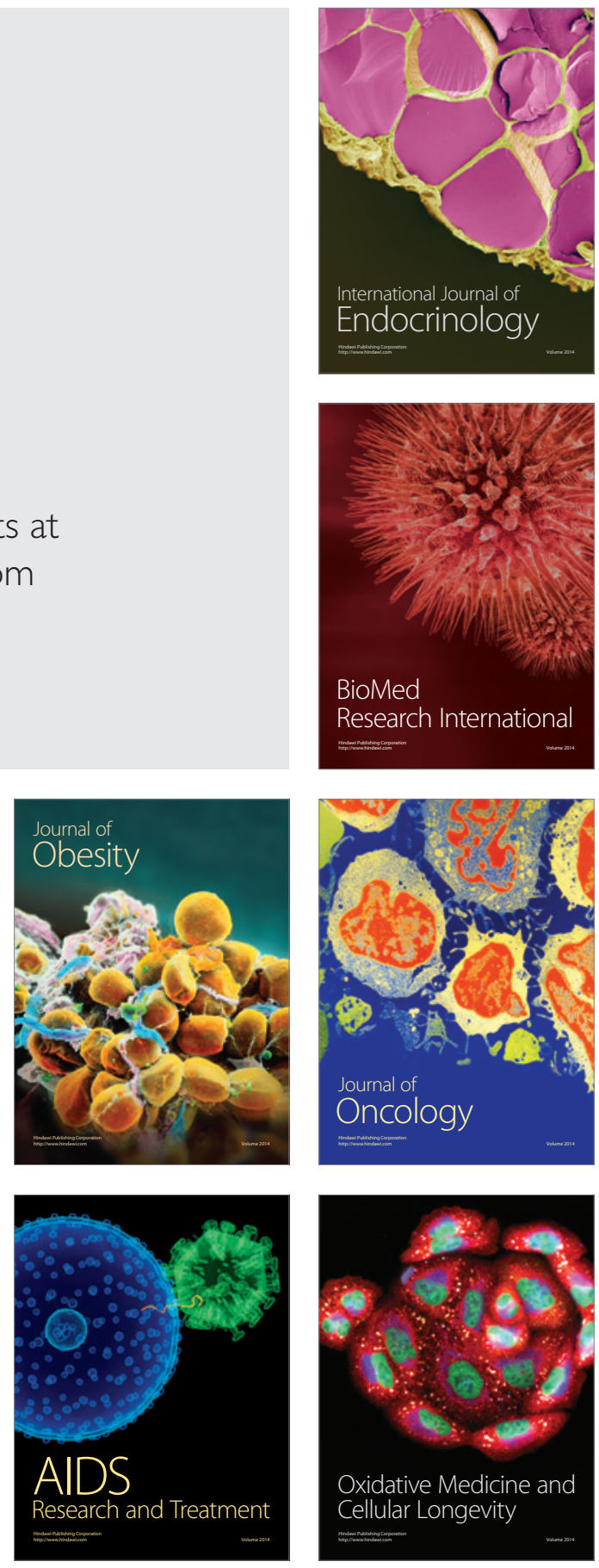